\title{
EXPERIMENTAL RESEARCH OF THE COUETTE FLOW WITH CROSS FLOW
}

\author{
Matthias NOBIS, Peter STÜCKE, Marcus SCHMIDT•
}

\begin{abstract}
When a solid cylinder is rotating inside a hollow cylinder, a characteristic fluid flow occurs inside the gap between the two cylinders, caused by the adhesion of the fluid at the walls. This flow problem is widely known as the Couette-flow. If an additional flow entrances through a radial located feedhole at the outer hollow cylinder, there is an interaction between the cross flow and the Couette-flow. In result there are complex three dimensional flow structures in the gap at the area around the feedhole.
\end{abstract}

These arising flow structures are closely related with the technical important flow inside the gap of hydrodynamic lubricated journal bearings. When the flow conditions inside the bearing gap are well explored and appreciated, it will be possible to give suggestions for constructive details like the design, the location and the dimension of the feedhole for longer lifecycles or an even more efficiently running.

In this paper the test rig of the bearing model will be presented. Moreover some representative results from researches with a Laser-Doppler-Velocimeter (LDV) in comparison with the output of three dimensional numerical simulations will be illustrated.

\section{INTRODUCTION}

The Taylor-Couette apparatus is a typical experimental setup for basic research in fluid dynamics. The apparatus described in presented work consists of a fixed outer cylinder and a rotating inner cylinder, shown in Figure 1 . The gap between the two cylinders is filled with a Newtonian fluid, e.g. water or silicon oil. Flow structures inside the cylindrical gap are characterised by the Reynolds number based on the rotational speed of the inner cylinder, the mean cylinder gap width and the viscosity of the fluid. The so-called Couette flow, which remains stable below a critical Reynolds number, is similar to the flow of the oil inside the gap of hydrodynamic journal bearings. A medium term objective is the application of the three-dimensional numerical flow simulation (3D-CFD) in geometrical setups of real journal bearings under usage of an adapted cavitation model. So it will be possible to get established information about the reasons and the consequences of cavitating oil in the small gap of journal bearings. To have knowledge about the precision and the reliability of the numerical simulations, it is necessary to compare and validate the 3D-CFD with experimental results.

\footnotetext{
- M.Sc. Matthias Nobis, email: matthias.nobis@fh-zwickau.de, phone: +49 375/536 3804

Prof. Dr.-Ing. Peter Stücke, email: peter.stuecke@fh-zwickau.de, phone: +49 375/536 3444

M.Sc. Marcus Schmidt, email: marcus.schmidt.1@fh-zwickau.de, phone: +49 375/536 3893
}

Westsächsische Hochschule Zwickau, Dr.-Friedrichs-Ring 2a, 08056 Zwickau, Germany 
The most important differences between a traditional Taylor-Couette apparatus and a real journal bearing are the considerable smaller gap width and a superimposed cross flow at the journal bearing. The pictures 2 and 3 in Figure 1 show, that the primarily assembly has to be expanded to a system with a radial feedhole in the outer cylinder. A larger inner cylinder leads to a smaller gap width. However, by transferring relevant fluidic parameters from the journal bearing to the test rig, it is possible to simulate the flow pattern in the gap of a real journal bearing.

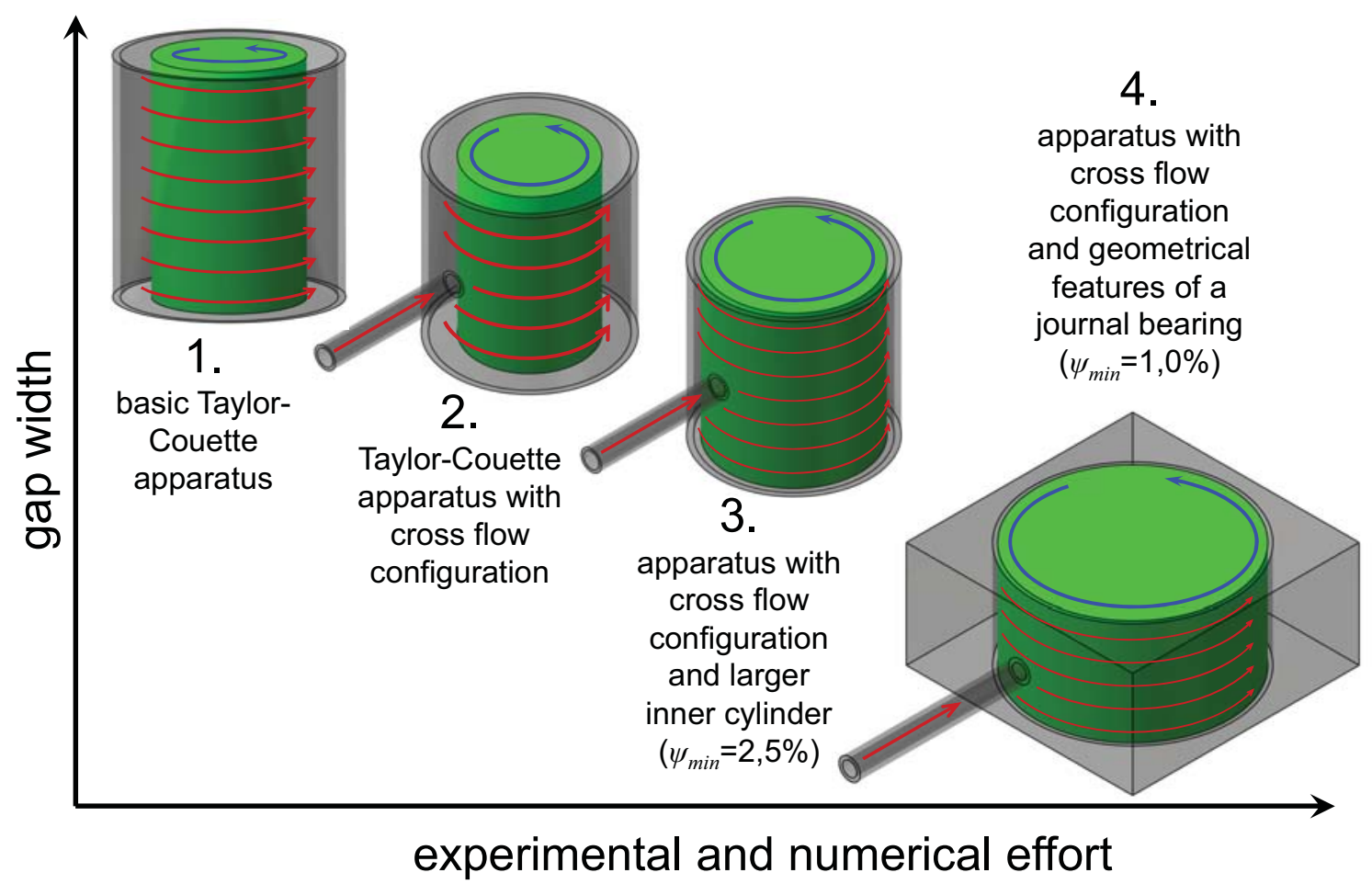

Figure 1: Experimental setups in an overview

The four pictures in Figure 1 are arranged in a diagram, which shows the gap width over the experimental and numerical effort. The higher experimental effort results from a more elaborate positioning of the LDV inside the gap and a more elaborate adjusting of the eccentricity, caused by the need of a higher accuracy by a system with a smaller gap width. The higher numerical effort results from a more complex volume mesh. The number of cells will increase, because the volume mesh has to be finer in the smaller gap maintaining the number of cells across the gap at the lowest tolerable number. Thus, the grid generation and also the simulation require more time. First of all the numerical simulation is validated in comparison to experimental measurements at a moderate gap width. In the context of this article, results of a system with a relative gap width of $10 \%$ will be presented. For further works there is projected an assembly like the picture 4 in Figure 1. Specified explanations are announced in the chapter conclusion and future works.

\section{Geometrical PARAMETERS}

A bearing system can be described simplified by a fixed outer cylinder and a rotating inner cylinder. The eccentricity is given by the displacement $e$ between the two cylinder centre axes. Orthogonal to the outer cylinder, in the middle of the breadth $B$ there is a 
feedhole for supplying the system with fresh fluid. The Figure 2 shows the according geometrical setup.
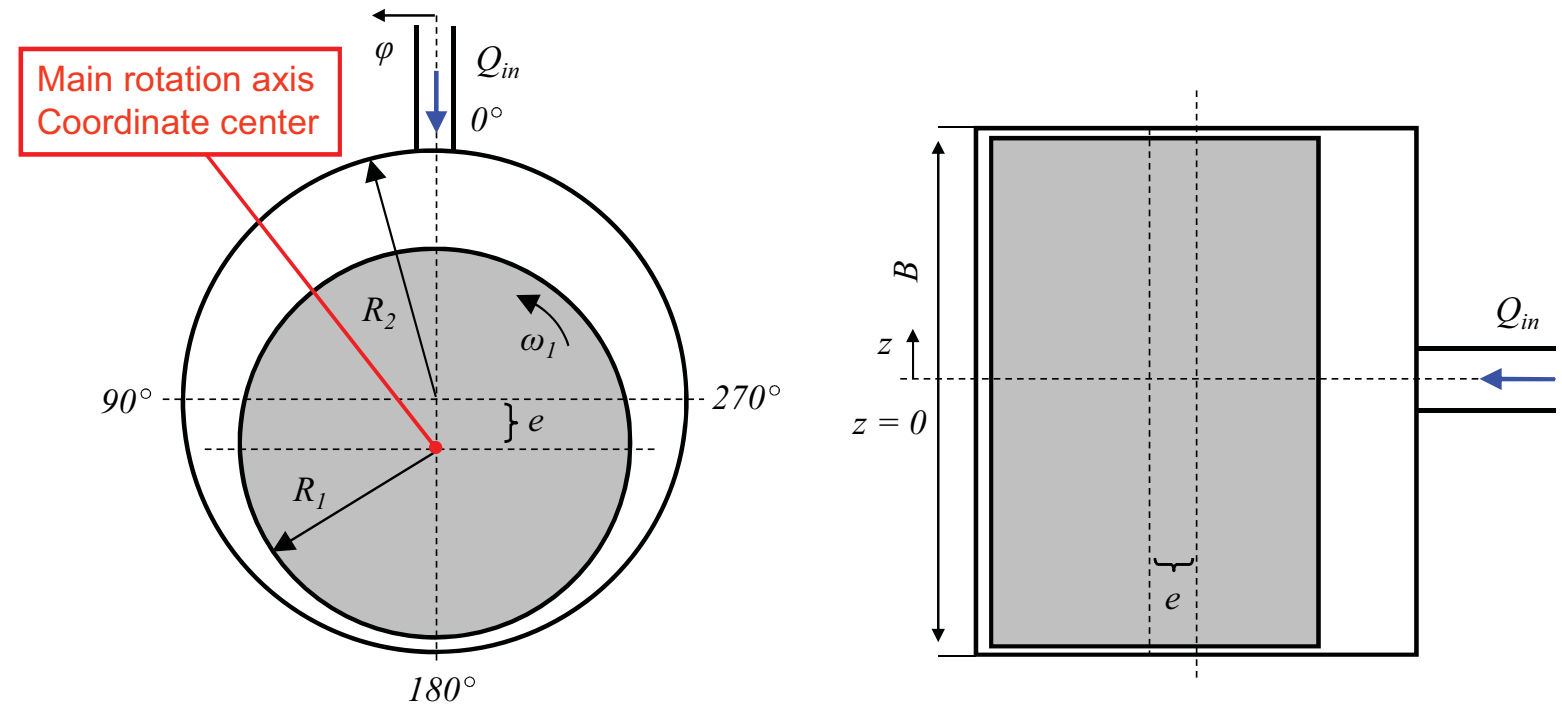

Figure 2: Geometrical parameters

The typical Reynolds number range for automotive applications was carried out in a previous work [6] and is between 10 and 40. The equations 1 till 8 define characteristic parameters.
$H_{0}=R_{2}-R_{1}$$$
Q_{0}=0,5 \cdot B \cdot H_{0} \cdot U_{1}
$$$$
\Psi=\frac{H_{0}}{R_{1}}
$$$$
\alpha=\frac{Q_{i n}}{Q_{0}}
$$$$
\Gamma=\frac{B}{H_{0}}
$$$$
\varepsilon=\frac{e}{H_{0}}
$$$$
U_{l}=\omega \cdot R_{l}
$$$$
R e=\frac{H_{0} \cdot R_{1} \cdot \omega}{v}
$$

\section{THE EXPERIMENTAL AND NUMERICAL SETUP}

The experimental apparatus consist of the fixed outer cylinder, which can be positioned eccentric in opposite to the rotating inner cylinder. There are radial mounted measuring screws at the outer cylinder to adjust a targeted eccentricity. The central point of the system is the driving shaft, where the inner cylinder is mounted and fixed with a screw nut. To have the opportunity to vary the nominal gap width and the relative bearing width, there are various inner cylinders with equal sizes. At present it is possible to realise a relative gap width of $\psi=2,5 \%$ or $\psi=10 \%$, which is defined by the diameter of the chosen inner cylinder. By operating with such a moderate relative gap width, it is possible to perform velocity measurements with a LDV. The LDV is connected to a traverse system and so the measuring volume of the LDV can be positioned very exactly inside the gap. To measure at various angle positions the turntable with the mounted outer cylinder can be rotated stepwise around the driving shaft. For applying the superimposed cross flow to the radial feed hole, there is a circuit with an adjustable oil pump and a storage tank. 


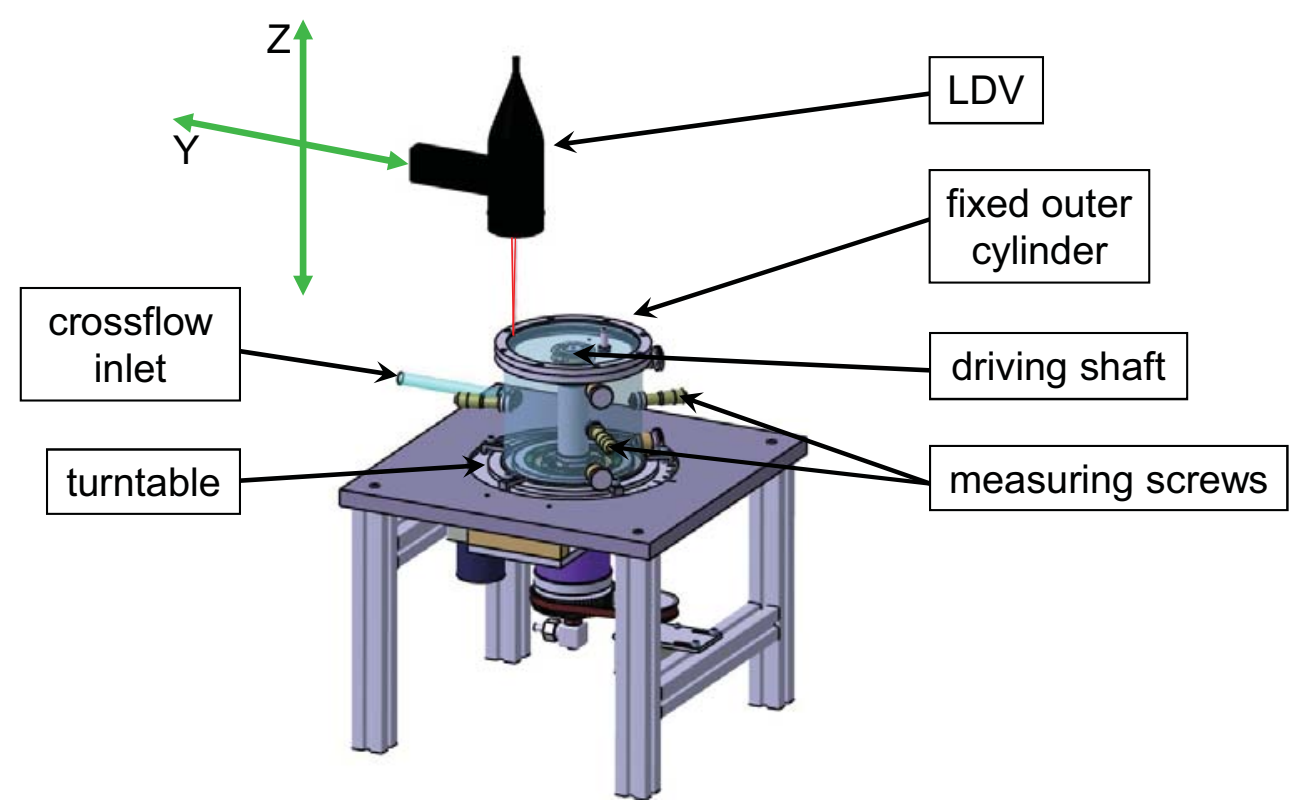

Figure 3: The experimental setup

To ensure an exactly dimensioned cross flow and a symmetrical outflow over the two axial boundaries of the system, variable area flow meters are in use (not pictured in Figure 3).

For the CFD simulation the experimental setup is transferred into a three-dimensional numerical model, which is shown in Figure 4. The calculation is done with the open source code OpenFOAM, which is a three-dimensional CFD code, based on the finite volume method. The used numerical equations are the Navier-Stokes equations and the continuity equation for incompressible, stationary flow.
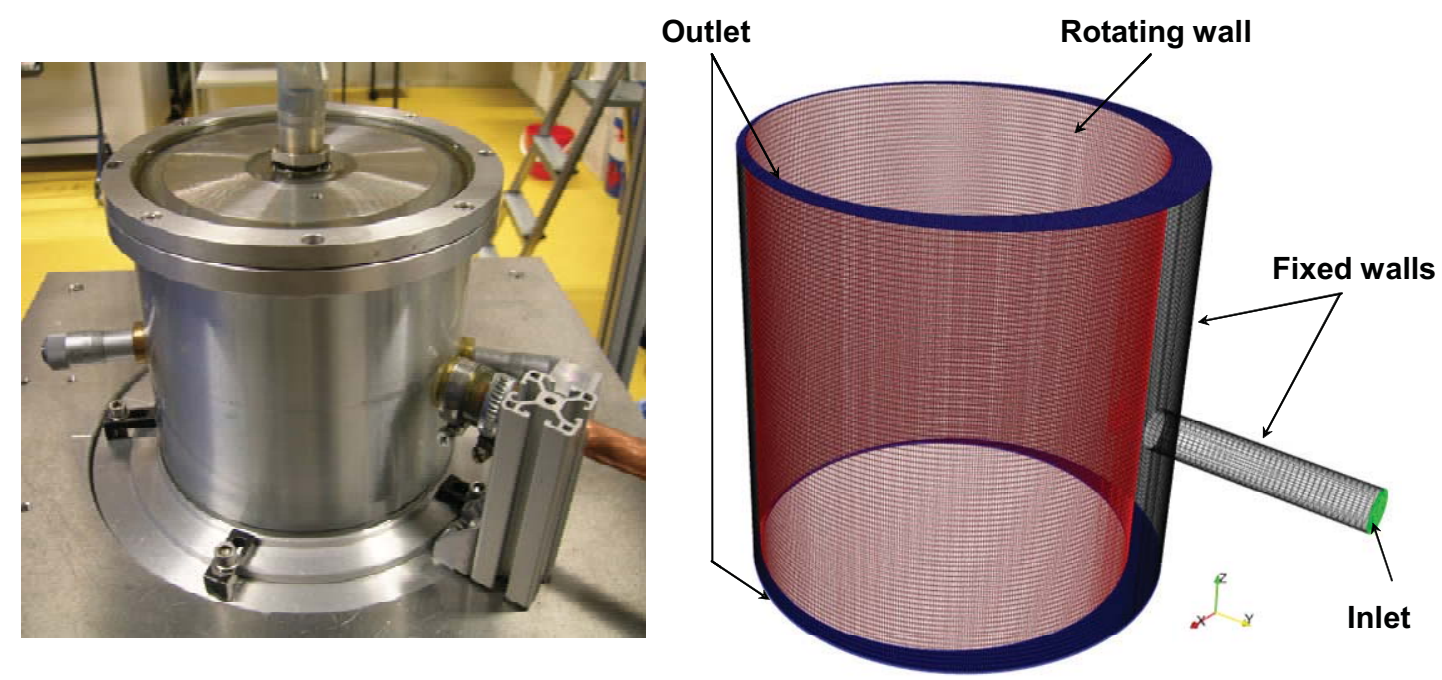

Figure 4: Experimental apparatus and numerical model

The numerical grid is generated as a block structured mesh with nearly orthogonal hexagonal cells. Especially the number of cells over the gap is important for the reproduction of the velocity profiles over the local gap width. 


\section{RESULTS OF RESEARCH}

The present velocity profile is generated about 20 sampling points in the vicinity of the feedhole over the local gap width at an angle of $\varphi=10,6$ (Figure 5). In this case the LDV is positioned in axial direction directly over the gap (compare Figure 3 ) and measures the circumferential velocity.

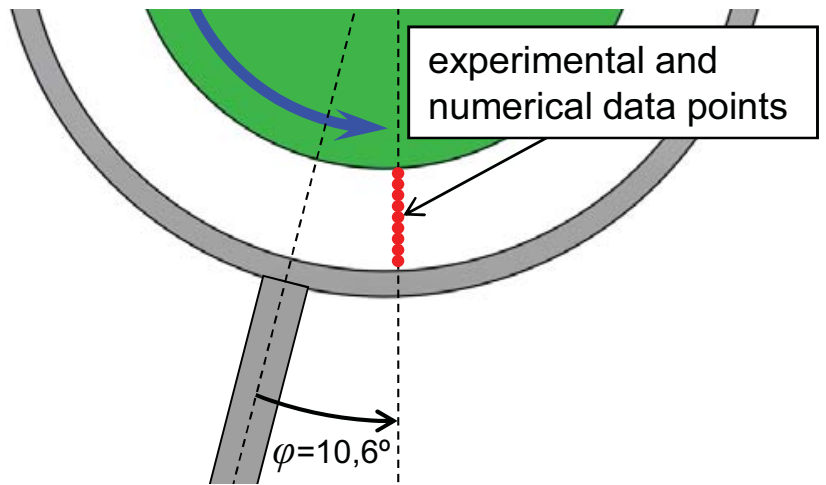

Figure 5: Sampling points

The graph with the triangular marks in Figure 6 shows a real measured velocity profile under the specified boundary conditions. The upper respectively the lower frame of the diagram could be understand as the surface of the rotating inner respectively the fixed outer cylinder.

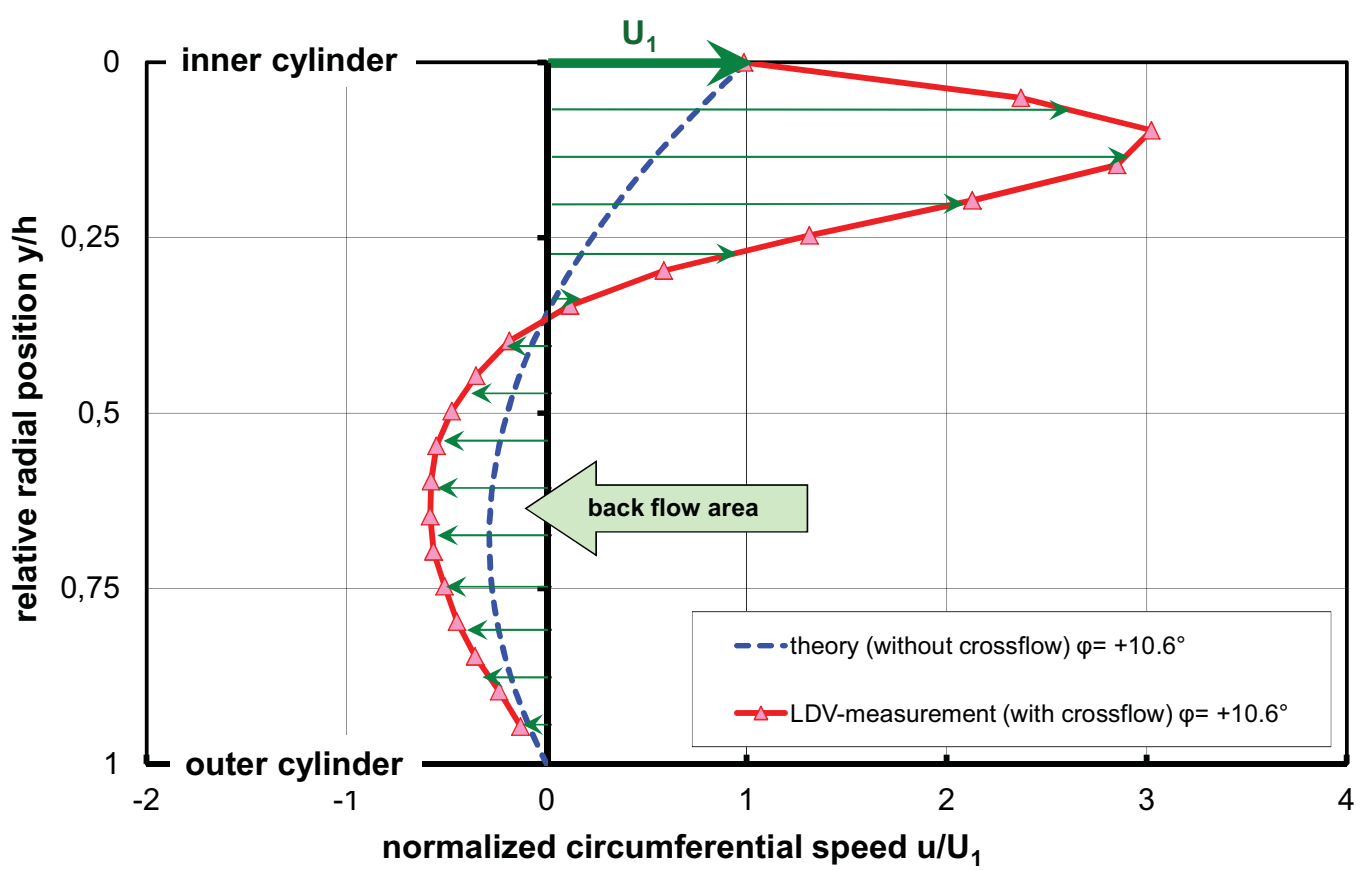

Figure 6: Velocity profile for $\psi=10 \%, \varphi_{B}=0^{\circ}, \operatorname{Re}=20, \alpha=100 \%$ and $\varepsilon=90 \%$ Regarding to the adhesion between the oil and the walls, directly at the surface of the inner cylinder the circumferential velocity $U_{l}$ will be measured. Consequently the normalized circumferential speed $u / U_{1}$ amounts one. At the fixed outer cylinder the velocity is zero. It is interesting to compare the theoretical profile, which is illustrated with the dotted line, and the experimentally obtained velocity profile. The dotted line can be calculated analytical for the basic Couette flow without any cross flow. Initial point for the calculation are the Navier-Stokes equations. Under usage of some simplifying 
assumptions, a squared equation can be developed, which delivers the normalized velocity by a previous specification of the normalized geometrical parameters. But the equation does not consider to any cross flow. Therefore the effects from the cross flow to the Couette flow becomes clearness. Near the surface of the inner cylinder, the flow is getting an enormous acceleration. The velocity of the flow in this area is three times higher than the velocity of the inner cylinder surface. Towards the wall of the outer cylinder the natural back flow area is accelerated. In this zone the fluid flows against the rotational direction of the inner cylinder.

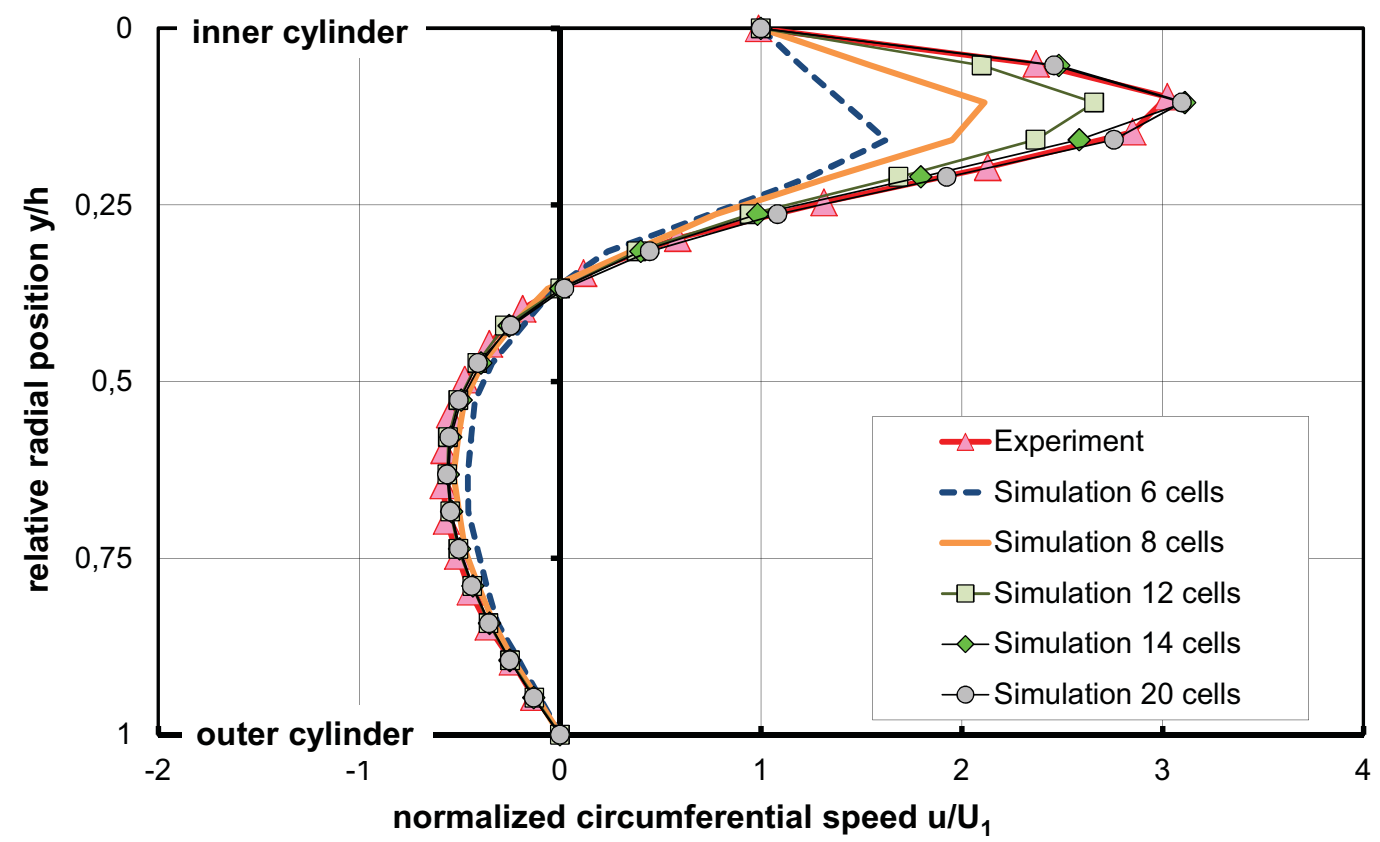

Figure 7: Comparison between experimental and numerical determined velocity profiles for $\psi=10 \%, \varphi_{B}=0^{\circ}, \operatorname{Re}=20, \alpha=100 \%$ and $\varepsilon=90 \%$

Figure 7 shows the result of a mesh test. The cell number over the gap is increased stepwise, to reach a mesh independent solution. If the cell number is too low, the numerical computation cannot reproduce the high velocity gradient near the surface of the inner cylinder. For a cell number of 14 over the gap the numerical solution is in perfect agreement to the LDV measurement. More cells would not improve the computation quality. There is also no difference between the solutions of the computation with 14 or 20 cells. This mesh test shows, that in the radial direction over the gap a cell number of 14 leads to a mesh independent solution. Hence, the overall cell number in the model amounts to 700000 .

\section{CONCLUSION AND FUTURE WORKS}

The explained test rig and the LDV measuring method enable the research of the velocity distribution inside cylindrical gaps with a moderate width. The showed proceeding is not applicable to a system with a gap width like a real journal bearing. One aim is to build a new test rig with larger dimensions and to reduce the relative gap width from actual $\psi=2,5 \%$ to $\psi=1 \%$. The nominal gap width $H_{0}$ stays compared to the presented setup constant and thus the LDV can be used furthermore. Plane outline faces like the picture 4 in Figure 1 make it possible, to measure from the radial direction with controllable refractions of the laser beams. 


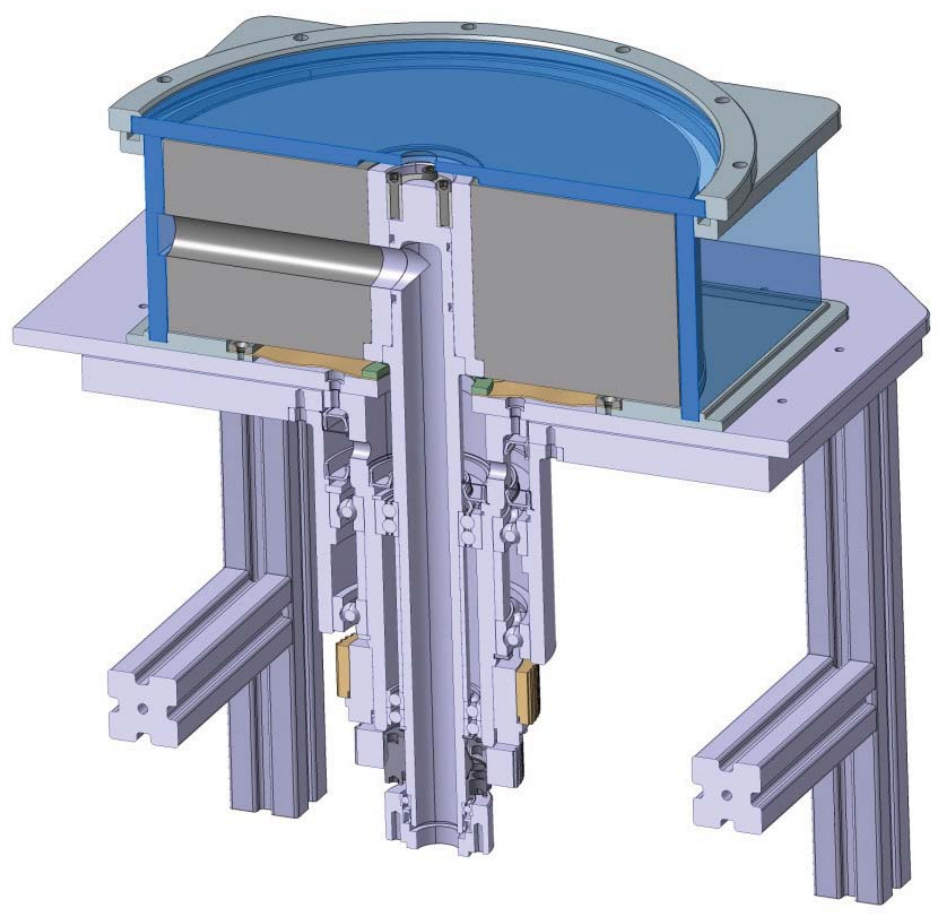

Figure 8: Improved test rig

Moreover, the rebuild of the test rig will include geometrical attributes, which can be found in the main bearings of the crankshaft from internal combustion engines. For example there will be a groove in the bushing and a hole for the outflow trough the inner cylinder. The two axial outlets for the applied cross flow will be retained. The fluid, which flows through the hole of the inner cylinder, will be conducted through the hollow driving shaft and a rotary union at the lower end of the shaft.

\section{NomenCLATURE}

$B \quad$ nominal bearing width

$e \quad$ eccentricity

$H_{0} \quad$ nominal gap width

$h \quad$ local gap width

$Q_{0} \quad$ inner volumetric flow rate

$R_{1} \quad$ radius inner cylinder (shaft)

$R_{2} \quad$ radius outer cylinder (bushing)

Re Reynolds number

$u \quad$ circumferential speed

$U_{1} \quad$ circumferential speed of the inner cylinder

$\alpha \quad$ ratio from applied volumetric flow rate to inner volumetric flow rate

$\Gamma \quad$ relative bearing width

$\psi \quad$ relative gap width

$\varepsilon \quad$ relative eccentricity

$v \quad$ kinematic viscosity

$\varphi \quad$ angle

$\varphi_{B} \quad$ angle of the feedhole position

$\omega$ angular velocity of the inner cylinder 


\section{REFERENCES}

[1] Taylor G. I.: Stability of a viscous liquid contained between two rotating cylinders, London, Phil. Trans. Royal Soc., 1923

[2] DiPrima R. C., Swinney H. I.: Instabilities and Transistion in Flow Between Two Rotating Cylinders, in Hydrodynamic Instabilities and Transistion to Turbulence, New York, (Hrsg.: H. I. Swinney and J. P. Gollup), Vol. 45, Springer, 1985

[3] Eagles P. M., Stuart J. T., DiPrima R. C.: The Effects of Eccentricity on Torque and Load in Taylor-Vortex Flow, Journal of Fluid Mechanics, Vol. 87, pp. 209-231 1978:

[4] Koschmieder E. L.: Taylor vortices between eccentric cylinders, The Physics of Fluids, Vol. 19, 1976

[5] Scurtu N., Stücke P., Egbers C.: Numerical and experimental study of the flow in an eccentric Couette-Taylor system with small gap, PAMM, Vol. 8, Issue 1, pp 10641 - 10642, 2008

[6] Stücke P., Nobis M., Schmidt M.: 3D-Flow Structures in Journal Bearings, San Antonio, Texas, SAE Powertrains Fuels and Lubricants Meeting, 2009

[7] Stücke P., Nobis M., Schmidt M.: Three Dimensional Flow Structures in Journal Bearings, West London, UK, 2nd Micro and Nano Flows Conference, 2009

[8] Stücke P., Nobis M., Schmidt M.: Numerical meshing issues for threedimensional flow simulation in journal bearings, Thessaloniki, Greece, 3rd Micro and Nano Flows Conference, 2011 